\title{
La autoevaluación: una reflexión para la gestión de los centros educativos
}

\section{Self-evaluation: A Reflection for School Management}

Recibido 02 abril 2012 • Aceptado 01 mayo 2012 • Corregido 07 junio 2012

\author{
Mario Alberto Segura Castillo ${ }^{1}$ \\ mseguraster@gmail.com \\ División de Desarrollo Curricular \\ Ministerio de Educación Pública
}

\begin{abstract}
Resumen. A continuación se presenta un ensayo acerca de cómo la convivencia, desde la autoevaluación de la gestión de los centros, es replanteada en la medida que el docente y el estudiantado dialogan acerca del papel que cumple la función mediadora en el aprendizaje en donde se diagnostica las dificultades y facilidades que tiene la población estudiantil, el personal docente y la gestión administrativa para desarrollar procesos orientados a lograr un mayor aprendizaje. Se concluye que la autoevaluación de la gestión institucional es un proceso sistemático de autorreflexión de los componentes endógenos y exógenos de la institución educativa, por parte de las instancias participantes a partir de un sinnúmero de propósitos de la gestión administrativa del centro y su plan de mejora.
\end{abstract}

Palabras Claves. Autoevaluación, interculturalidad, centros educativos y convivencia

Abstract. This is an essay about how coexistence and living from self-management of schools. Teachers and students dialogue about the role played by mediation strategies in learning where they diagnosed difficulties and facilities that have the student population, teachers and administrative management to develop processes aimed at achieving greater learning. As a conclusion, self-assessment in institutional management is a systematic process of self-reflection with endogenous and exogenous components of same educational centers, by participating institutions from purposes of school management and its improvement plan.

Keywords. Self-evaluation, intercultural elements, educational and living, coexistence.

1 Doctor en Mediación Pedagógica de la Universidad La Salle. Máster en Evaluación Educativa de la Universidad de Costa Rica (UCR). Maestría en Democracia y Valores de la Universidad de Barcelona, España. Licenciatura en Educación con énfasis en Administración Educativa de la Universidad de Costa Rica (UCR) y Bachillerato en la Enseñanza de los Estudios Sociales de la Universidad de Costa Rica (UCR). Actualmente se desempeña como Asesor en la Dirección de Desarrollo Curricular del Ministerio de Educación Pública de Costa Rica. Ha publicado artículos en diferentes revistas en el campo de la educación. 


\section{Un acercamiento a la autoevaluación de centro educativos}

La autoevaluación se ocupa de la reflexión en los procesos de construcción de conocimientos, competencias sociales y afectivas así como habilidades psicomotoras potencialmente significativas para la persona. Esto se construye por medio de un proceso de socialización que permite maximizar las potencialidades de la persona. Es importante destacar que en el aprendizaje sistematizado las personas involucradas aprenden mejor cuando fijan metas.

En el documento "El Centro Educativo de Calidad como Eje de la Educación Costarricense", el Ministerio de Educación Pública (MEP, 2008) indica que:

Se considera que la educación debe partir desde la situación cognoscitiva de la individualidad del estudiantado, sus intereses e idiosincrasia, de sus respectivas estructuras de conocimiento ya formadas y a partir de ellas emprender la acción formativa y promover el aprendizaje como proceso dinámico, siempre va a estar sujeto a las posibilidades de perfeccionamiento y de una constante evolución. (p.14)

Es evidente entonces, que se asuma la autoevaluación como un proceso en el cual la persona construye, de manera socializada, integral y activa, nuevas ideas o conceptos basados en conocimientos presentes y pasados, para aplicarlos en la resolución de problemas y en la interpretación de situaciones nuevas, por lo cual, es importante la participación y colaboración de otras personas. Asimismo, potencia la metacognición por medio de los procesos de mediación, los cuales generan secuencias de eventos que permiten la construcción de saberes y la correspondiente adaptación de las estructuras de pensamiento. La autoevaluación, también promueve la exploración libre de la persona dentro de un contexto o de una estructura previamente definida, que adquiere mayor nivel de complejidad respondiendo a sus etapas evolutivas por medio de la participación y de la intervención de la comunidad educativa en una comunicación permanente de forma asertiva, respetuosa y afectiva.

Se colige como un proceso de perpetua construcción mediante relaciones interpersonales, acordes con las características, los intereses y las necesidades de las 


\section{Q⿳⺈⿴囗十⺝⿱⺈⿻コ一心 \\ Escuela de Administración Educativa}

[Número publicado el 30 de Junio del 2012]
Revista Cientifica Digital ISSN:2215-2288

URL:http:// revista digita l.ea e.fc s.uc r.ac.cr/

personas, según su edad y el contexto sociocultural al que pertenecen. En este proceso el personal docente se constituye en promotor de oportunidades pedagógicas y aprendiz, en tanto orienta, aprende y facilita experiencias que promueve la creatividad, el gozo, la curiosidad y el razonamiento necesarios para la obtención y aplicación de los aprendizajes en las distintas situaciones de la vida.

La construcción colaborativa de los conocimientos facilita que la autoevaluación en el salón de clases sea lúdica, que las relaciones interpersonales sean afectivas y que se produzca la apropiación individual de los conocimientos de acuerdo con el nivel de desarrollo intelectual, psíquico y social del estudiantado.

\section{Hacia una cultura autoevaluadora en la convivencia}

Para Morin (2003, p. 30) la "educación debe fortalecer el respeto por las culturas, y comprender que ellas son imperfectas en sí mismas, como lo es el ser humano". Por su parte, se plantea la incorporación del pensamiento complejo en la educación para facilitar a la generación de una política compleja que permita desarrollar la complejidad desde la doble pareja pensar global/actuar local, pensar local/actuar global.

El aporte fundamental del pensamiento complejo a la formación de la persona es entender en los educandos el desarrollo del pensamiento, para enfrentar los grandes problemas que hoy sufre la humanidad que requiere una nueva dimensión de ciencia holística y sistémica: "los problemas que implican incertidumbre e imprevisibilidad, interdependencias e interretroacciones de extensión planetaria relativamente rápida, con discontinuidades no lineales, desequilibrios, comportamientos caóticos y bifurcaciones" (Morin, 2003, p. 136).

Por lo tanto, la tarea de educación es crear un espacio de transformación en el convivir de las personas, para vivir. Esta necesidad de saber, de búsqueda de un conocimiento que nos permite interrelacionarnos, conectarnos, de consensuar la comunicación eficiente, y participar en procesos de aprendizaje, es lo que se suele identificar con el término de pedagogía, la cual surge entre las personas que integran la comunidad educativa bajo la idea de respeto recíproco de sus derechos, con el fin de enfrentar el reto educativo.

Asimismo, según Morín (2003) los centros educativos en la actualidad forman parte del proceso de la globalización, que busca 


\section{Cabstion}

[Número publicado el 30 de Junio del 2012]
Revista Cientifica Digital ISSN:2215-2288

URL:http:// revista digita l.ea e.fc s.uc r.ac.cr/

[...] homogenizar lo que se aprende; por ejemplo, qué se compra, qué se enseña, incluso en qué persona escribimos (voz pasiva): La lógica que los preside es la de competición en todos contra todos y todas y la de evadir responsabilidades. La educación tiene que ver con la complejidad como salto cualitativo clave para ayudar a salirnos del estado pavoroso en el que nos sumergieran hasta asfixiarnos las odiosas separaciones de las ciencias y cuyas fragmentaciones también han mutilado una y otra vez a nuestra propia humanidad. (p.46)

Lo anterior, se relaciona con la promoción de espacios de participación ciudadana efectivos, legítimos y representativos, donde la educación sea responsabilidad de todas las instancias sociales, por lo que en este proceso de homogenización es importante considerar la promoción de una cultura de la autoevaluación en los centros educativos, la cual no busca cuantificar los procesos pedagógicos y administrativos, sino investigar el progreso de la acción educativa que se lleva a cabo y su incidencia en el desarrollo y construcción de una sociedad aprendiente y sostenible, por ello, interesa más el para qué evaluar, que el qué evaluar o el cómo evaluar.

Es importante comprender la cultura de autoevaluación con la convivencia, la cual fomenta la discusión de ideas desde diferentes sectores, sobre el aporte de la educación en el desarrollo del país y la inserción del mundo con conciencia planetaria, con la potenciación de procesos para la toma de decisiones derivadas de la investigación, la reflexión, la evaluación y el consenso con la participación de la ciudadanía. Por lo anterior, es importante considerar la autoevaluación como estrategia en la gestión del centro educativo:

- La vinculación de la gestión administrativa con la gestión pedagógica de forma integrada.

- El diseño de iniciativas que fomenten la discusión de ideas desde diferentes sectores, sobre el aporte de la educación para el desarrollo del país.

- La promoción de procesos para la toma de decisiones derivadas de la investigación, la reflexión y el consenso con la participación de la comunidad educativa y todas las instancias sociales. 


\section{G Gestión \\ Escuela de Administración Educativa}

[Número publicado el 30 de J unio del 2012]
Revista Cientifica Digital ISSN:2215-2288

URL:http:// revista digita l.ea e.fc s.ucr.ac.cr/

Mediante los procesos de autoevaluación, desde la gestión de los centros educativos, se fortalece el respeto por las culturas, y se comprende que ellas son imperfectas en sí mismas, como lo es el ser humano. Cada cultura merece vivir una vida digna, justa de aceptación y responsabilidad con las demás, para que se produzca la resonancia mórfica y labremos un mundo más pleno, espiritual y feliz.

Asimismo, es importante crear una cultura de autoevaluación en los centros educativos, debido a los procesos que conllevan o no hacia la convivencia, por ejemplo: la deserción y la exclusión en todas las modalidades y niveles del sistema educativo, por lo que se torna importante el diseño del currículo centrado en las personas, teniendo en cuenta las potencialidades, necesidades, intereses y las características del nivel de cognoscitivo en que se encuentran, así como los contextos donde se desenvuelven. "La autoevaluación institucional es una necesidad no suficiente sentida y ejercitada, Cada profesor actúa en su aula y se preocupa, fundamentalmente, de la evaluación de sus alumnos" (Santos, 1995, p.133).

También, considerar en los procesos de la cultura de autoevaluación en el centro educativo, el establecimiento de procesos de articulación y transición en los primeros años de cada ciclo, para la adaptación del currículo a las características del desarrollo del estudiantado, tomando en cuenta la metodología, la relación docente-estudiante, los planes y programas de estudio, así como la infraestructura educativa. Es de suma importancia la creación de una cultura de autoevaluación institucional que integre aspectos formativos, diagnósticos y sumativos, para la valoración integral del estudiantado y de los procesos de la gestión de los centros educativos.

Desde la cultura de autoevaluación sería conveniente el diseño de estrategias para que el estudiantado y el profesorado desarrollen metodologías atractivas, retadoras y coherentes con las características de ritmos y estilos de aprendizaje de la población estudiantil los cuales propicien el pensamiento crítico y el disfrute de los procesos de mediación y de aprendizaje significativo.

Con el afán de democratizar el currículo es necesario la transformación de los centros educativos en comunidades de aprendizaje colaborativo, que se caractericen por promover ambientes agradables, seguros, participativos y favorables para la toma de decisiones mediante una cultura autoevaluadora de sus prácticas y rutinas cotidianas. 


\section{Gẹcestión}

[Número publicado el 30 de Junio del 2012]
Revista Cientifica Digital ISSN:2215-2288

URL:http:// revista digita l.ea e.fc s.ucr.ac.cr/

Con los procesos de la cultura de autoevaluación se promueve la incorporación de distintos canales de acceso y salida del conocimiento, tales como actividades deportivas, artísticas, culturales, lúdicas y recreativas que hagan más atractivo, retador e interesante el proceso educativo, favoreciendo la atención a la diversidad y por ende, el éxito y permanencia del estudiantado en el centro educativo. Aunado a lo anterior, es necesario que en los procesos de autoevaluación de la gestión del centro se construyan estrategias educativas acordes a las necesidades y características de poblaciones específicas, tales como: estudiantes talentosos, grupos extra edad, personas que han sido privadas de libertad, jóvenes que trabajan, madres adolescentes, adultos, niñas, niños y jóvenes en situaciones de violencia intrafamiliar, migrantes, personas con necesidades educativas especiales, con discapacidad, grupos indígenas, entre otros.

El centro educativo y la familia podrían lograr procesos autoevaluativos conjuntos para la detección temprana de situaciones de riesgo, de este modo elaborar medidas de prevención y protección de la población estudiantil.

\section{El aporte de la autoevaluación al currículo en la formulación de un Plan de Mejora}

Por "currículum se entiende a la síntesis de elementos culturales (conocimientos, valores, costumbres, creencias, hábitos) que conforman una propuesta político - educativa pensada e impulsada por diversos grupos y sectores sociales cuyos intereses son diversos y contradictorios" (De Alba, 1994, p.38).

El currículo es concebido como la integración sistemática de todos los componentes que inciden en el quehacer educativo, para el desarrollo integral de la persona y de la sociedad, en armonía con la naturaleza y consecuente con un desarrollo sostenible. Visto así, el currículo es una propuesta intencional que se ampara en las respectivas legislaciones del país y se programa en forma consensuada para cumplir con los fines y con los objetivos de salida del sistema educativo, debido a que el currículo provee una formación sólida en el dominio de los saberes, capacidades, valores y actitudes.

La autoevaluación de la gestión del centro propicia un currículo costarricense caracterizado por ser: "contextualizado, humanizante, inclusivo, intercultural, versátil, dinámico, participativo, interdisciplinario, flexible, práctico, transversal, reflexivo, horizontal, 


\section{Giestion: \\ Escuela de Administración Educativa}

[Número publicado el 30 de Junio del 2012]
Revista Cientifica Digital ISSN:2215-2288

URL:http:// revista digita l.ea e.fc s.ucr.ac.cr/

descentralizado, procesual, sistemático y coherente; de manera que atienda armónicamente la formación de las personas que el país requiere" (UNESCO, 2010, p.13).

De acuerdo con la UNESCO (2010, p.13) el currículo en el Sistema Educativo Costarricense "permite la identificación de los problemas y desafíos del contexto en el que se desenvuelve la persona, los factores culturales que deben conservarse y los que necesitan transformarse para la toma de decisiones educativas y curriculares".

Esta concepción de currículo resulta dinámica, en tanto se sustenta en la interrelación de sus diferentes componentes, lo que implica es lograr procesos de la cultura de autoevaluación para la comprensión de las relaciones que se establecen entre la institución educativa y la comunidad, la legislación actual, los programas de estudio, las metodologías, los recursos, el contexto escolar, comunal y global, el cuerpo docente, el estudiantado y la familia que demandan de un Proyecto de Mejora construido con la participación de todas las instancias educativas.

El currículo en ningún caso representa un producto final acabado, más bien refiere a un proceso en construcción. Así, asumido de esta manera, transforma el quehacer pedagógico, buscando que se adapte a la realidad propia de las instancias participantes en el proceso educativo.

La autoevaluación introduce al currículo a una perspectiva dinámica de la diversidad cultural. También la propuesta se centra en el contacto y la interacción. De ahí, que Rojas (2007, p.50) resalta "La influencia mutua, el sincretismo, esto es, en formas de interacción sociocultural cada vez más intensas y variadas en el mundo cambiante, debido a la globalización económica, política, ideológica y a las innovaciones en comunicación y transportes".

Asimismo, "la escuela presenta las condiciones fundamentales para preparar al estudiantado para los procesos de aceptación y valoración de las variables culturales, más allá de las alternativas asimilacionistas o compensatorias y muy vinculadas a formulaciones educativas afines de la educación inclusiva" (Rojas, 2007, p.70).

Existen diversas razones para justificar los procesos autoevaluativos en el currículo, debido a la responsabilidad de los centros educativos de desarrollar procesos de aprendizaje que respondan a las diferencias individuales desde los elementos culturales que la persona aprendiente lleva consigo. También, desde un punto de vista social, considerando los 


\section{Giestion: \\ Escuela de Administración Educativa}

[Número publicado el 30 de Junio del 2012]
Revista Cientifica Digital ISSN:2215-2288

URL:http:// revista digita l.ea e.fc s.ucr.ac.cr/

procesos de aprendizaje de la niñez, la adolescencia y del adulto puede cambiar sus actitudes frente a la diferencia y ser el andamio para una sociedad más justa, no discriminatoria y la construcción de valores como la tolerancia, solidaridad, el respeto y el amor o sea la convivencialidad.

La cultura de autoevaluación permite caracterizar y organizar internamente los elementos del currículo se fundamentan epistemológicamente en el humanismo. Desde el enfoque humanista, el propósito de la educación es la formación integral de la persona y su autorrealización, propicia y potencia al máximo su desarrollo en relación con el contexto de pertenencia, lo que implica el cultivo del amor por sí mismo, por sus semejantes, hacia las diversas formas de vida que existen en el planeta, el desarrollo de un sentido ético y estético, así como la participación en la vida ciudadana dentro de un sistema democrático.

Es importante, la implementación de un currículo integrado, con la formulación de cualquier diseño curricular centrado en la persona y en los aspectos de trascendencia de cualquier sistema educativo, en términos de horas lectivas, jornadas diarias de trabajo, variedad de asignaturas, recursos didácticos y recursos humanos especializados, bibliotecas escolares, infraestructura, comedores escolares, acceso a la tecnología, contextualización de contenidos y de metodologías a la realidad de los centros educativos, principalmente en zonas rurales. La ampliación de las ofertas educativas para las zonas rurales de manera que se atienda a la población estudiantil acorde con las particularidades del contexto.

La autoevaluación permite la revisión de las estrategias utilizadas para la inserción de la población que no se le han dado las oportunidades pertinentes en el sistema educativo, a los diferentes grupos etáreos en los distintos procesos de alfabetización, así como, el desarrollo de procesos de actualización, asesoramiento y capacitación con personal técnico y docente que atiende la población de personas jóvenes y adultas en el sistema educativo.

El diseño de un modelo curricular centrado en la persona y en los aspectos de trascendencia para el país, exhorta a un fortalecimiento de los diversos ciclos y modalidades educativas con la finalidad de un mejoramiento de la gestión administrativa vinculada con el desarrollo del currículo:

- Vinculación de la gestión administrativa con la gestión pedagógica de forma integrada. - La articulación de una gestión administrativa que atienda el mantenimiento y la 


\section{QGestión \\ Escuela de Administración Educativa}

[Número publicado el 30 de Junio del 2012]
Revista Cientifica Digital ISSN:2215-2288

URL:http:// revista digita l.ea e.fc s.uc r.ac.cr/

reparación de la infraestructura educativa, que permita la atención a la diversidad y el mejoramiento de la calidad de la educación.

- Fortalecimiento de programas de compensación social y desarrollo de una gestión administrativa adecuada de estos, disminuyendo los trámites administrativos que obstaculizan la atención oportuna de las necesidades del estudiantado.

La autoevaluación centra la discusión curricular en contenidos que versan por sí mismos una relevancia social, por lo que es de suma importancia una visión interdisciplinar mediante ejes problemas, se discuta y se tomen decisiones en cuanto a la conservación de recursos básicos para la subsistencia humana (agua, suelo, energía, aire, biodiversidad). La promoción de un desarrollo integral de la sexualidad, teniendo en cuenta edad y desarrollo evolutivo. Así como la erradicación de estereotipos de género y se diseñen iniciativas que erradiquen las manifestaciones de cualquier tipo de violencia, como el uso de armas, drogas lícitas e ilícitas, abuso y explotación sexual, y embarazo adolescente. En otras palabras se fomente los estilos de vida saludables y la felicidad de las personas que se forman en el sistema educativo.

Asimismo, es importante que se tomen decisiones fundamentales en cuanto a la integración de las tecnologías de la información y la comunicación (TIC) en el currículo, es importante considerar los siguientes temas de discusión:

- Desarrollo de las competencias para el uso de las tecnologías que requiere el personal docente y técnico docente.

- Correcta utilización de la información digital para la construcción de conocimientos y aprendizajes significativos.

- Apoyo administrativo, pedagógico y técnico necesario para que el estudiantado forme parte en la sociedad de la información y el conocimiento.

- Aplicación de un enfoque pedagógico apropiado.

- Acceso a los recursos tecnológicos actuales y apropiados, tanto en equipo como en programas y conectividad.

La cultura de autoevaluación hoy día promueve en los centros educativos y en el 
contexto comunal la convivencia armónica entre diferentes grupos humanos, el respeto y enriquecimiento entre los diversos grupos éticos que comparten el mismo espacio, que de acuerdo con León y García (2008) indican:

Si aceptamos este reto, deberíamos entender que la educación va más allá del reconocimiento y respeto a la diversidad étnica, deberíamos colaborar en la construcción de un centro educativo que entendiese las diferencias humanas como necesarias y enriquecedoras tanto para el desarrollo colectivo como individual. (p.3)

Lo anterior, es la concepción de un centro educativo inclusivo e intercultural, que a nuestro juicio debe cumplir las siguientes características:

- Entender la cultura del otro como un conjunto de valores que pueden enriquecernos tanto como nuestra propia cultura.

- Valorar positivamente la diversidad cultural como elemento imprescindible para resolver conflictos multiculturales.

- Reconocer el derecho a la propia identidad cultural.

- Abordar la implicación de una educación intercultural como paso necesario para el desarrollo de una sociedad global.

- Desde adentro se puedan romper con todas las barreras de aprendizaje que obstaculiza que la población estudiantil aprenda, por consiguiente, reciban una educación de calidad.

Desde dicha visión anterior, han surgido los planteamientos de la autoevaluación, la cual busca transformar el ambiente escolar para que a todo el estudiantado se le reconozca sus derechos como persona, en reconocimiento de su pertenencia a un determinado grupo etario, sin ningún tipo de exclusión, para lo cual es importante que los centros educativos como instituciones rompan todas las barreras que limita el aprendizaje de la población estudiantil, tal y como se ha visto, promover valores dentro y fuera del centro educativo con la plena participación de todas las instituciones comunales y la familia para que se realice el diálogo y la convivencia, para que realmente se dé el ejercicio de la ciudadanía mediante procesos de autoevaluación en el centro educativo. 


\section{Gestión: \\ Escuela de Administración Educativa}

[Número publicado el 30 de Junio del 2012]
Revista Cientifica Digital ISSN:2215-2288

URL:http:// revista digita l.ea e.fc s.uc r.ac.cr/

Para Bonil, Sanmartí, Tomás, y Pujol (2004)

la equidad orienta una acción que, sin caer en el relativismo cultural, asume el respeto a la vida en todas sus manifestaciones. Entiende que cada individuo comparte el mundo con los demás, y ello está intrínsecamente unido a la aceptación de la alteridad y la pluralidad. La apuesta por la equidad comporta la necesidad del autoconocimiento y la autoaceptación de los individuos y de las colectividades, así como el conocimiento y la aceptación de los demás. (p.8)

Desde el punto de vista de la autoevaluación se reconoce que dentro del quehacer educativo todos son interculturales y esto exige tomar conciencia de los valores y creencias como parte de los procesos de enseñanza y aprendizaje. Se representa todas las dimensiones que de una o de otra manera afectan el sistema educativo y los principios fundamentales del currículo que se relacionan directamente con la interculturalidad, desde los siguientes puntos de vista:

- La educación representa una construcción cultural llevada a cabo en ámbitos desde diversos referentes culturales.

- La diversidad enriquece los escenarios educativos, debido a la convivencia de diversos referentes culturales.

- Se favorece a la construcción continúa desde el diálogo de las diversas culturas.

- Se asume la diversidad como una norma, todo grupo humano es diverso culturalmente y puede ser descrito en función de sus características culturales.

- La educación es un derecho humano fundamental, por tanto, toda cultura tiene dicho derecho, para una convivencia más justa.

La educación debe prestar atención a aquellos estudiantes que tradicionalmente han sido excluidos de las oportunidades educativas, por ejemplo: el estudiantado con necesidades educativas especiales, discapacidades, con capacidades superiores, migrantes, la niñez pertenecientes a minorías étnicas y lingüísticas, entre otros. 


\section{Gegesion:}

[Número publicado el 30 de Junio del 2012]
Revista Cientifica Digital ISSN:2215-2288

URL:http:// revista digita l.ea e.fc s.ucr.ac.cr/

\section{La autoevaluación en la mediación pedagógica}

La persona es una unidad conformada por cuerpo-mente-espíritu, dotada de una red interminable de interconexiones e interdependencias con el medio donde se desenvuelve, y que gracias a ellas crea su conciencia, al igual que sus relaciones con la colectividad que la rodea y a donde promueve el desarrollo de su inteligencia.

A la vez que se da el aprendizaje espontáneo, existen maneras que facilitan, disponen, acercan el conocimiento a los aprendientes. Estas son: la mediación, la pedagogía, las tecnologías de información y comunicación que evidentemente transforman la manera de aprender.

Desde la cultura de autoevaluación, la mediación pedagógica es definitivamente un puente que entrelaza y conecta el conocimiento con el proceso de aprendizaje. Toda actividad didáctica es mediada desde el contenido para hacer de esta actividad una, llena de sentido y por ende, llena de significado para el que aprende.

Por esta razón, el proceso de mediación debe contemplar todos los factores que favorezcan y promuevan aprendizajes con sentido y de calidad. Cada instante, cada situación, cada persona es una fuente inagotable de aprendizaje y a la vez, se convierte en el objeto de estudio de cada uno de los aprendientes, pues encontrará en ellos una y mil preguntas y querrá una y mil respuestas.

La mediación pedagógica parte de la necesidad de promover experiencias de aprendizaje, de lograr la interlocución y la interactividad con estudiantado, de comprender que en la autoevaluación da lugar a lo imprevisible, de la necesidad de compartir para construir juntos la creación del saber como un proceso científico, indagatorio y lúdico, busca que las actividades, estrategias, ejercicios y procedimientos de los tratamientos biopedagógicos se conviertan en experiencias de aprendizaje placenteros, significativos y novedosos que permiten el ser en el hacer, en convivencia, en perpetuo diálogo. Además, la persona disfruta del conocimiento y de los procesos de aprendizaje, donde se promueve el desarrollo de su capacidad de asombro e imaginación, como llave para entrar al mundo del conocimiento y a la construcción de saberes, de valores y de actitudes positivas frente a la vida, lo que se vincula con la experiencia cotidiana y la realidad social, cultural y económica de la persona aprendiente, de manera que la autoevaluación se torna significativa y pertinente para el personal docente. 
En consecuencia, el gran reto que se plantea la cultura autoevaluadora es lograr activar ese emocionar en cada uno de los aprendientes, es conseguir que estos vuelquen su atención al descubrimiento de lo nuevo, de lo contrario no hay aprendizaje. En otras palabras es proporcionar retos, es sensibilización, es ofrecer situaciones que permitan la incorporación de nuevos hallazgos, es el caos, es la incertidumbre, es un reacomodo y un nuevo orden. Igualmente importante resulta el ordenamiento de los currículos escolares para responder a esta complejidad, a esta trama de la vida. Si el diseccionar la realidad para ser el objeto de estudio, causa problemas lineales y de poca integralidad curricular, lo imperativo es atravesar el universo de los saberes mediante un sistema transdisciplinar, transversal que permita la comprensión y el estudio de la realidad de la manera más representativa.

El estudiantado y el profesorado se transforman juntos mediante la convivencia, a través de relaciones e interrelaciones, en la biología del amor, reflexionando y respetándose mutuamente. Ambos acuden al centro educativo acarreando sus propias realidades, su presente y su pasado propios; pero también, ambos son partes fundamentales del devenir histórico, genético, cósmico y evolutivo de la humanidad. Bien lo dice Maturana (1996), los seres vivos son el presente de esa historia de cambio, por lo que creemos fervientemente, que nuestros estudiantes y nuestros profesores deben educarse en la cultura sostenible del cambio, la cual debe fomentar que lo único permanente en él es precisamente el cambio.

A lo largo de los planteamientos anteriores, la cultura de autoevaluación implica el reconocimiento de la existencia de diferentes puntos de partida, la interconectividad entre los saberes y la ausencia de verdades permanentes. Este último aspecto es importante valorarlo debido a que la evolución de los descubrimientos en los distintos campos del saber produce la renovación continua del conocimiento, mediante los procesos de mediación pedagógica. El personal docente clarifica la propuesta metodológica con que se desarrollará la asignatura, garantiza que las pautas que se incluyan reflejen las líneas pedagógicas y metodológicas que han sido asumidas para el diseño de la propuesta curricular para la formación integral. Las pautas que se propongan en el salón de clases deben considerar los siguientes principios o premisas:

- Lo afectivo se reconoce como el elemento más importante en los procesos de aprendizaje. 


\section{Gegetion}

[Número publicado el 30 de Junio del 2012]
Revista Cientifica Digital ISSN:2215-2288

URL:http:// revista digita l.ea e.fc s.uc r.ac.cr/

- La actividad lúdica forma a la persona en relación con las demás y consigo misma.

- La construcción cooperativa de los conocimientos facilita su apropiación individual (proceso interior progresivo y diferenciado que es preciso respetar)

- Cada persona construye sus propias herramientas conceptuales y su propio aprendizaje.

- La individualización permite reconocer las diferencias en la diversidad.

- La vinculación de las estrategias pedagógicas con la experiencia cotidiana y la realidad social, cultural y económica de la persona.

\section{Interrelaciones en la cultura de la autoevaluación}

Desde la conciencia ecológica la cultura de autoevaluación se convierte en conocer nuestra condición humana unida a la naturaleza, por lo tanto, se caracteriza por entender las emociones, sentimientos y valores de la población estudiantil, personal docente y administrativo bajo el principio de responsabilidad por la vida digna y justa, generando el efecto mariposa y compartir esperanza al concienciarse de las pasiones de los demás y sentirlas como propias, construyendo para la vida que habita y es la pequeña Gaia.

El propósito básico de la autoevaluación es definido como una tarea necesaria para reorientar procesos, llamar la atención sobre casos excepcionales, dinamizar el proceso de formación integral, buscar estrategias más conducentes y operativas. Asimismo, la autoevaluación de la gestión institucional es un proceso sistemático de autorreflexión de los componentes endógenos y exógenos de la institución educativa, por parte de las instancias participantes (personal docente y administrativo, estudiantes, padres de familia y personas e instituciones de la comunidad), a partir de la valoración de los objetivos, misión y visión institucionales.

Es importante entender la gestión del centro educativo desde la totalidad para poder construir procesos autoevaluativos. La totalidad se entiende mirando las partes, a la vez de ser consciente de los nexos indisolubles con el todo, desde el origen de la humanidad, el ser humano lo contempla desde totalidades que contienen el universo en uno. Al respecto, Serra, Iriarte y Le Fosse (2000) indican que: 


\section{Qigestión: \\ Escuela de Administración Educativa}

[Número publicado el 30 de J unio del 2012]
Revista Cientifica Digital ISSN:2215-2288

URL:http:// revista digita l.ea e.fc s.ucr.ac.cr/

Los sistemas no lineales son aquellos que generan respuestas que no se comportan en forma directa y proporcionada cambios en alguna variable, sino que se derivan de la trayectoria previsible. Los sistemas complejos son aquellos que están compuestos por muchas partes. Los sistemas dinámicos son aquellos cuyo estado depende del momento en que son observados. (p.67)

Dentro de los principios de un sistema dinámico, complejo, no lineal y estocásticos, se encuentran:

- Sinergia: El todo es mayor a la suma de sus partes. El valor de un sistema se reduce cuando se divide en sus componentes individuales.

- Conectividad: Todo sistema vivo es la suma de interconexiones indivisibles, que se forman de maneras no lineales.

- Identidad: Los sistemas se organizan alrededor de una idea central, la que proporciona una identidad fuerte y un sentido de propósito que trasciende los cambios estructurales.

- Equilibrio dinámico: Los sistemas vivos establecen un balance dinámico, en donde van fluctuando dentro de determinados parámetros estables buscando un equilibrio.

- Creatividad: Los sistemas vivos mutan en forma permanente. La habilidad de formar nuevos niveles de orden es la verdadera naturaleza de la creatividad.

- Apertura: La creatividad de los sistemas se incrementa por tres motivos: a) los agentes del sistema interactúan en forma intensiva tanto internamente como externamente, b) la información es rica, diversa y fluye libremente, c) existe una tremenda diversidad en los agentes de un sistema.

- Flexibilidad: Estos sistemas son flexibles y de gran plasticidad, debido al proceso de autoorganización que se produce en sus estructuras para adaptarse a las condiciones del medio ambiente. (Serra, Iriarte, Le Fosse, 2000, p. 68)

El pensamiento complejo y la convivencia persuaden acerca de la responsabilidad del actuar y accionar en un sitio determinante, incide en la estructura del planeta y del universo, de la misma forma que el actuar por pequeño que parezca para bendecir y dar vida, puede 
representar la iniciativa para que la totalidad de seres humanos quieren actuar y salvar el planeta.

La cultura, sociedad y lenguaje son atributos y distintivos de lo humano, inseparables, dinámicos, autocreadores, autorreferentes. En esta trinidad cada sujeto individual es un punto del holograma que contiene el todo de la humanidad (Morin, 2003).

Cada vez es más evidente la existencia de una sociedad y una naturaleza múltiple y compleja que muestra diversidad de visiones del mundo, todas ellas desarrolladas a partir de la representación que cada colectivo posea, como producto de su cultura, de las experiencias y el pensamiento compartido por medio del lenguaje.

Se necesita una sociedad global con conciencia de comunidad de destino planetario, una cultura que herede la ecología mental o noosfera, para posibilitar como parte de la naturaleza, con ella y que dependemos unos de otros, por lo cual se debe tratarnos dentro de la naturaleza y a ella dentro de nosotros.

Es importante redimensionar nuevos pactos entre todos los seres humanos, para alcanzar la sociedad global sostenible, lo que refiere Boff (2006):

Es fundamental, el papel que juega el lenguaje, para lo cual se deben establecer símbolos, que nos permitan una visión colectiva, y promover el pensamiento, la conciencia de que somos una sola familia humana y una sola comunidad con un destino común, la cual necesita elaborar un proyecto planetario solidario y una gestión colectiva de los problemas, con objeto de conferir sostenibilidad a la vida del Planeta. (p.87)

En convivencia es posible crear una sociedad, en cuyo interior se elabore el proyecto de desarrollo, porque el proyecto mismo no subsiste por sí mismo. Se sustenta en la sociedad fundada en el respeto hacia la naturaleza, los derechos humanos, la justicia económica y una cultura de paz que promueva la búsqueda del bien común, la autolimitación y la justa medida. Para lograrlo es imperativo un cambio de mentalidad y de corazón (UNESCO, 2000).

La autoevaluación, es precisamente la respuesta a redimensionar la pobreza, desde la dimensión humana y ecológica, partiendo de premisas fundamentales de lo que esta significa, convirtiéndola en el sujeto principal de su propia educación, mediante, el respeto a su cultura, a la capacidad de colaboración, de organización de su propia capacidad de autodeterminarse para la solidaridad con el otro y el encuentro de la persona con su propia libertad en una 
sociedad más justa, fraternal y ecológicamente integrada. "En el bioaprendizaje es preciso enfatizar las interconexiones entre los seres humanos, los fenómenos sociales y los naturales" (Gutiérrez y Prado, 1997, p.17).

Para Boff (2006), en el pasado no se redimensionaron las competencias que tienen los pobres para solidarizarse y luchar por la injusticia para liberarse con la ayuda de las tres ecologías. Así, por ejemplo, desde la ecología ambiental, las personas tenemos la capacidad de ser responsables de proteger el hábitat, indudablemente promover desde cada uno de los espacios relacionales en los que nos desenvolvemos, acciones atenienses al cuido de la comunidad con el objetivo primordial de preservar la biodiversidad existente, en armonía con el ambiente, como medio de conservar la dinámica ecológica.

Para cumplir con todo esto, es imperativo que en convivencia aprendamos a conocernos y a respetarnos, a ser responsables de nuestras acciones individuales y aquellas de nuestros congéneres, con quienes compartimos en sociedad, responsabilidades como ciudadanos del mundo, amantes de la paz, la igualdad, la solidaridad y la fraternidad.

Esto exige que todos los miembros de la sociedad humana han de familiarizarse con el sistema ético y de valores de la sociedad sostenible, con el fin que sean el motor de cambio en la civilización; uno de los aspectos fundamentales es que la autoevaluación tiene que ver con humanizar lo humano, el alma, la mente, es decir, con el espacio relacional o psíquico que vivimos y que deseamos que vivan la niñez y las personas adolescentes, para que la nueva cultura sea heredada a generaciones futuras más solidarias y sororales.

Estas nuevas relaciones tienen que ver con el respeto y la cooperación, con la armonía biológica y cultural; con la confianza y el placer de convivencia; con el afecto, la dulzura y el amor; con la participación, la flexibilidad y la solidaridad; con la admiración por la belleza y el misterio de la vida; con la unión y la veneración por la naturaleza; con la creatividad, el conocimiento intuitivo y la dimensión espiritual del ser humano (Gutiérrez y Prado, 1997, p.63).

Desde el aprendizaje se piensa en una nueva alianza entre los seres humanos con la naturaleza, se requiere un nuevo diluvio universal, un nuevo compromiso desde las ecologías: ambiental, social y espiritual. Dicha alianza con "la Gaia, es el superorganismo vivo y con todos los seres que en él existen y viven, mediante relaciones nuevas de benevolencia, compasión, 


\section{Gesestibn: \\ Gìn Educación \\ Escuela de Administración Educativa}

[Número publicado el 30 de J unio del 2012]
Revista Cientifica Digital ISSN:2215-2288

URL:http:// revista digita l.ea e.fc s.uc r.ac.cr/

solidaridad cósmica y profunda veneración por el misterio que cada cual porta y revela. Solo entonces habrá una liberación integral del ser humano y de la Tierra" (Boff, 1996, p.147).

Es importante recordar, que nuestro origen es común con todo lo que existe en el universo, somos parte del mismo todo y por tanto, las plantas, aves, animales, rocas, estrellas, soles, son nuestras hermanas y hermanos y nosotros como seres humanos.

\section{Consideraciones finales: Entornos de la autoevaluación}

Desde el autoevaluación existe un problema de responsabilidad social de la evaluación de los aprendizajes y el estado de la educación, debido a que en educación no todo es cuantificable, ni medible, ni conviene que lo sea. (Segura, 2007). Lo más importante de una persona es lo que no se puede medir. Desde la autoevaluación propone que haya dentro de los centros educativos espacios para el diálogo y la reflexión de todas las instancias que participan de los procesos educativos de la población estudiantil.

El propósito básico del evaluador es definido como una tarea necesaria para reorientar procesos, llamar la atención sobre casos excepcionales, dinamizar el proceso de formación integral, buscar estrategias más conducentes y operativas.

La autoevaluación de la gestión institucional es un proceso sistemático de autorreflexión de los componentes endógenos y exógenos de la institución educativa, por parte de las instancias participantes (personal docente y administrativo, estudiantes, padres de familia y miembros de la comunidad), a partir de la valoración de los objetivos, misión y visión institucionales. Lo que conlleva

Considerar la (auto) evaluación como instrumento de reflexión y análisis de la práctica, así como de desarrollo profesional e institucional. Ambos procesos, (auto) evaluación y mejora, son dos caras de una misma moneda, lo que significa que deben estar íntimamente relacionados. La (auto) evaluación, por tanto, debe estar orientada a la mejora efectiva de la práctica educativa, lo que supone una actitud, disposición y habilidad para legitimar en términos de valor las propuestas de cambio, ajustarlas a las propias situaciones y contextos, y reconstruirlas desde la acción reflexiva y crítica En la 
práctica la autoevaluación debe constituirse más en una filosofía, que en una estrategia. (Murillo, 2008, p.5)

La autoevaluación de las formas de proceder fija su atención en los procesos en sí mismos, incluso al margen de los resultados obtenidos. Es evidente que podemos evaluar en términos de resultados los actos agresivos manifiestos en la conducta del estudiantado, pero resultaría muy difícil evaluar la agresividad solo a partir de este sistema, por lo tanto, no trata de cuantificar, sino de investigar el progreso de la acción educativa que se lleva a cabo y su incidencia en el desarrollo y construcción de los objetivos institucionales, por ello, interesa más el para qué evaluar, que el qué evaluar o el cómo evaluar.

Para autoevaluar la implementación de programas y proyectos en la institución educativa se sugiere usar los indicadores críticos que corresponden a los indicadores observables. Estos permiten identificar y descubrir comportamientos específicos que estén claramente relacionados con los objetivos finales que se desea lograr en función de cada de los programas y proyectos, de acuerdo con el contexto del centro educativo. Las actitudes que se seleccionen como indicadores críticos deben corresponder a aquellas actividades esenciales que marcan claras diferencias entre lo planificado y lo actuado, deben ser consensuadas por el conjunto de profesores, padres de familia y administrativos.

La autoevaluación requiere un proceso sistemático que implica aplicar principios, métodos e instrumentos que permitan aumentar la objetividad. Los métodos y técnicas de evaluación dependen del sentido y la forma que se dé a la evaluación, deben ser confiables y válidos, deben reunir el punto de vista de los profesores administrativos y el estudiantado, debe contribuir al mejoramiento de la práctica docente, considerada como el verdadero contexto formativo en actitudes y valores.

Con la finalidad de analizar hasta qué punto un centro escolar cumple un mínimo de condiciones para implementar un Plan de Mejora (PM), se elabora una pauta para que el equipo de profesores realice el proceso de autoevaluación.

Entre los aspectos a tomar en cuenta se encuentran:

- Participa el personal docente y administrativo en forma crítica en todas las acciones del proceso de autoevaluación con base en la propuesta del PM. 


\section{Gestión : \\ G Educación \\ Escuela de Administración Educativa}

[Número publicado el 30 de J unio del 2012]
Revista Cientifica Digital ISSN:2215-2288

URL:http:// revista digita l.ea e.fc s.uc r.ac.cr/

- Cuenta la institución con personal motivado para el cumplimiento de los objetivos institucionales en el cumplimiento del PM.

- Se realizan las actividades propuestas en el PM durante todas las etapas del curso lectivo.

- Se cuenta con financiamiento adecuado para el cumplimiento de los proyectos propuestos en el PM.

- Existe disposición del personal docente y administrativo para elaborar, ejecutar y evaluar periódicamente el PM.

- Existen buenas relaciones humanas para la coordinación con las diferentes instancias participantes del proceso educativo (padres de familia, estudiantado y personal docente y administrativo).

La autoevaluación consiste en determinar las condiciones y los requisitos previos para iniciar bien la incorporación de los programas y proyecto. La autoevaluación permite a las instancias participantes observar el progreso de todos los elementos que intervienen en la ejecución del Proyecto de Mejora para evidenciar el proceso de cada una de las etapas y realimentar las áreas problemáticas. Se presenta como un testimonio vivencial de los educandos de manera individual o grupal, facilitando con ello, las posibilidades de compartir conocimiento e inquietudes sobre el proceso de mediación del aprendizaje, de construcción y reconstrucción de este.

Dentro de las características más relevantes de los Planes de Mejora, según Murillo (2008) son las siguientes:
a) Es una actividad voluntaria de los centros.
b) Se establece un compromiso del centro consigo mismo.
c) La actuación es compartida.
d) Los procesos de coordinación son fundamentales.
e) Importancia de un adecuado liderazgo.
f) Actuación relevante en los procesos que genera en los centros.
g) Asesoramiento y seguimiento interno y externo. (p.8) 
Lo anterior, se relaciona directamente con el concepto de la calidad educativa, el cual, combina la cobertura, la permanencia y los componentes básicos que permiten las acciones autoevaluadoras, con el fin de reconocer que es un desafío permanente que debe alcanzarse con equidad y que se construye paulatinamente, de allí la importancia de tomar en cuenta las particularidades nacionales y evitar toda expresión de exclusión. La búsqueda de la calidad en las instituciones educativas se realiza en la medida en que se coloque realmente a las personas en el centro de toda la actividad educativa. "La autoevaluación, como un proceso por el que la institución se mira a sí misma, tiene en su raíz una carga de coherencia importante: ¿Estamos consiguiendo lo que buscamos? ¿Estamos consiguiendo precisamente lo contrario? ¿Por qué?" (Santos, 1995, p. 133).

\section{Referencias bibliográficas}

De Alba, A. (1994). Currículum: crisis, mito y perspectivas. México: Universidad Nacional Autónoma.

Boff, L. (1996). Ecología: grito de la tierra, grito de los pobres. Madrid: Impresiones Trotta.

Boff, L. (1996). La dignidad de la tierra. Madrid: Impresiones Trotta.

Boff, L. (2006). Lección inaugural de la Universidad de Costa Rica. San José: UCR.

Bonil, T.; Sanmartí, J.; Tomás, C. y Pujol, R. M. (2004). "Un nuevo marco para orientar respuestas a las dinámicas sociales: el paradigma de la complejidad". Revista de Investigación en la Escuela, 53. Madrid, España.

Gutiérrez, F. y Prado, C. (1997). Ecopedagogía y ciudadanía planetaria. Heredia, Costa Rica: ILPC.

León, M. Á. y García B., M. (2008). Retos educativos de la escuela de nuestro tiempo: la interculturalidad. Madrid: CES - Don Bosco.

Maturana, H. (1996). El sentido de la humano. Santiago de Chile: Dolmen.

Murillo, P. (2008). "La autoevaluación institucional: un camino importante para la mejora de los centros educativos". Revista Organización y Gestión Educativa, 1, pp.13-17.

Ministerio de Educación Pública [MEP]. (2008). El centro educativo de calidad como eje de la educación costarricense. San José, Costa Rica: MEP.

Morin, E. (2003). El método V. La humanidad de la humanidad. Madrid: Colección Teorema Serie Mayor.

Rojas, G. (2007). Propuesta pedagógica en formación intercultural, fundamentada en principios y procedimientos de educación para la paz, para el aula preescolar de la escuela León XIII de Tibás. Tesis para optar por el grado de Maestría en pedagogía con énfasis en la Diversidad en los procesos educativos. Universidad Nacional de Costa Rica. Heredia, Costa Rica.

Santos, M. A. (1995). La evaluación: un proceso de diálogo, comprensión y mejora. España: Ediciones ALJIBE. 
Segura, M. (2007). "La perspectiva ética de la evaluación de los aprendizajes desde un enfoque constructivista". Revista de Innovaciones Educativa, 1. Universidad de Costa Rica.

Serra, R.; Iriarte, J. y Le Fosse, G. (2000). El nuevo juego de los negocios. Buenos Aires: Editorial Norma.

UNESCO (2000). La carta a la tierra: una declaración de principios fundamentales para la construcción de una sociedad global justa, sostenible y pacífica en el siglo XXI. París: WDE.

UNESCO (2010). Datos mundiales de la Educación en Costa Rica. 2010-2011. Paris: WDE. 